\title{
Fortificación de la harina de trigo con ácido fólico en Chile. Consecuencias no intencionadas
}

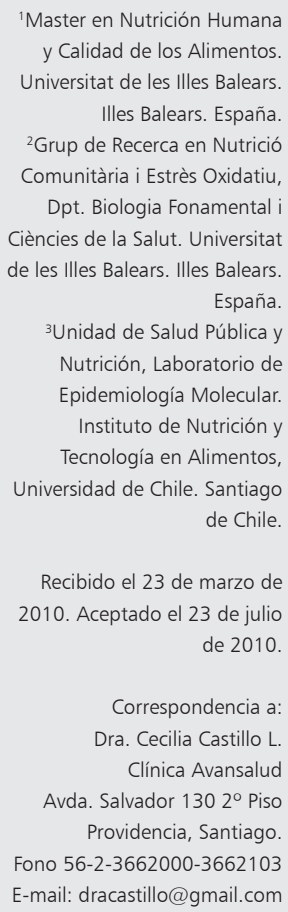

\author{
CECILIA CASTILLO L. ${ }^{1}$, JOSEP A. TUR ${ }^{2}$, RICARDO UAUY ${ }^{3}$
}

\section{Flour fortification with folic acid in Chile. Unintended consequences}

Background: High daily intake of folic acid (FA) could determine health risks in some populations. Aim: To review the Chilean FA wheat flour fortification and to identify the existence of populations at risk. Material and Methods: We categorized the FA levels in flour samples (percentil P) (2005-2008) and estimated intake of FA $(\mathrm{mg} / \mathrm{d})$ in adults from apparent bread consumption according to different levels (P20, 50 and 95) and children consumption (8-13 years) considering socioeconomic status (SES), bread/g/d intake (P20, 50 and 75) and regulated level of flour fortification (2.2 mg FA/100 g). Daily Dietary Folate Equivalent (DFE) consumption was estimated from serum folate in adults and elderly people (both sexes). We calculated the percentage of population with FA intakes over the estimated average requirement (EAR) and maximum level (UL) pre and post-fortification. Results: There is great variability in FA flour: 10-20\% samples without FA and 10-30\% with levels $>2.2$ mg/100 g. Adult daily consumption (2-4 day/loaves) could determine FA intakes close to UL. Children daily bread consumption (low socioeconomic level) > P75 have intakes close to UL. Post-fortification estimated daily DFE from serum folate in women, men and elderly people show: 99\% of women, 100\% of men and the elderly people have intakes higher than EAR. Additionally 2.3\% of women and 6\% of men would have intakes near the UL. Conclusions: The flour FA levels and serum folate levels in some populations show increased FA post-fortification intakes, which could lead to greater risk suggesting a revision of the fortification level.

(Rev Med Chile 2010; 138: 832-840).

Key words: Folates, folic acid, fortified food, risk assessment.

E n el año 1996, el Ministerio de Salud (MINSAL) de Chile desarrolló un estudio de carga enfermedad que mostraba que las malformaciones congénitas del recién nacido representaban 5,85\% de la carga total de enfermedad estimada, con altos costos asociados a su manejo, tratamiento y rehabilitación ${ }^{1}$.

Durante 1997, el MINSAL invitó a diversos sectores sociales a evaluar la factibilidad de implementar la fortificación masiva de la harina de trigo con ácido fólico (AF) como una estrategia para prevenir los defectos del tubo neural (DTN) del recién nacido (RN). Estos alcanzaban a 16/10.000 nacidos vivos y representaban un porcentaje importante de las muertes infantiles ${ }^{2}$ constituyendo cerca de $10 \%$ de las consultas del Instituto de Rehabilitación Infantil ${ }^{3}$.

La fortificación de la harina de trigo se estableció como obligatoria a partir de enero de 2000 (2,0-2,4 mg de AF/ $/ \mathrm{kg})^{3,4}$, siendo su principal objetivo aumentar la ingesta de folatos, especialmente en mujeres en edad fértil ( $400 \mu \mathrm{g} /$ día $)$ y reducir la prevalencia de los $\mathrm{DTN}^{5-8}$.

La harina de trigo fue seleccionada como ve- 
hículo para el AF porque el pan es un alimento de consumo diario en Chile ${ }^{9}$ y su adición no altera las características organolépticas del pan y derivados ${ }^{4}$. Esta intervención ha sido altamente costo-efectiva determinando una importante reducción de los DTN a través de los años $(40 \%)^{10,11}$.

Sin embargo, la existencia de una mayor oferta de alimentos fortificados ha generado preocupación a nivel internacional y un creciente interés por evaluar la existencia de riesgos asociados a su exceso en grupos que no se benefician con esta intervención ${ }^{12}$.

Estudios recientes muestran que una suplementación con ácido fólico efectuada antes del desarrollo de una neoplasia suprimiría su desarrollo y progresión pero favorecería su desarrollo cuando ésta existe ${ }^{13-19}$. El cáncer de colon, así como el cáncer de mama y próstata, podrían relacionarse con ingestas elevadas de folatos, especialmente, como ácido fólico. Se describen además anemia y alteraciones neurológicas en ancianos con ingestas elevadas de folatos ${ }^{16,17}$.

La mayor ingesta de ácido fólico se produciría por niveles de fortificación superiores a lo establecido y por mayor consumo de alimentos fortificados o suplementos nutricionales ${ }^{20}$ que determinarían una mayor probabilidad de exceder la ingesta máxima tolerable o "upper level" (UL) $)^{12,21,22}$.

Considerando estos antecedentes, en este estudio se analiza la fortificación de la harina de trigo con ácido fólico en Chile, con el objetivo de conocer la existencia de poblaciones en riesgo por ingestas excesivas de dicho nutriente.

\section{Material y Método}

\section{Fuente de datos}

La información sobre niveles de AF en harina se solicitó a la Autoridad Sanitaria: Departamento de Nutrición y Alimentos, Secretarías Regionales Ministeriales (SEREMIs) y al Instituto de Salud Pública (ISP) a través del portal Chile Transparente, vía e-mail o telefónicamente a los jefes de sección. La información del contenido de AF en muestras de harinas fue entregada por el ISP en un CD conteniendo archivos en planillas Excel (20052008). La información de normas y seminarios fue entregada en documentos Word y Power Point por el Departamento de Nutrición y Alimentos.
Los antecedentes de niveles de folatos sanguíneos se recopilaron de artículos chilenos y de información obtenida de algunos talleres efectuados en el MINSAL ${ }^{23-26}$. El consumo de pan estimado en niños fue extraído de una publicación nacional ${ }^{27}$.

\section{Análisis de datos}

La información sobre contenido de AF en harina fue categorizada en percentiles. Se estableció como población en riesgo aquellas con ingestas diarias de AF cercanas al UL $(600 \mu \mathrm{g} / \mathrm{d} /$ niños y $1.000 \mu \mathrm{g} / \mathrm{d} / \mathrm{adultos})^{21}$. Se calculó la ingesta de AF proveniente del consumo aparente de pan, considerando un contenido de $\mathrm{AF}$ en harinas según valores de los percentiles (P) 20, 50 y 95 de cada distribución anual. Para cálculos, se consideró un pan promedio de 100 gramos conteniendo 87 gramos de harina. El aporte de AF proveniente de otros alimentos no fue incluido en el análisis.

En población infantil se estimó ingesta de AF considerando el nivel promedio establecido en la norma de harinas $(2,2 \mathrm{mg} / \mathrm{kg})$ y el consumo de pan en gramos/día proveniente de una encuesta alimentaria efectuada en la Región Metropolitana, con datos estratificados por nivel socioeconómico (NSE), grupo de edad (8-9, 10-11 y 12-13 años), y percentiles de consumo (P25, 50 y 75) $)^{25}$.

Se aplicó la fórmula desarrollada por Quinlivan \& Gregory para determinar la ingesta total de folatos pre y post-fortificación considerando valores de folatos sanguíneos ${ }^{28,29}$. Esta fórmula describe la relación entre folatos séricos y consumo de folatos $(y=0,0145 x+0,132 r=-0,979 p<0,0001)$, siendo $\mathrm{x}=$ ingesta de ácido fólico e $\mathrm{y}=$ cambio $\mathrm{o}$ nivel de folatos séricos como equivalentes dietarios de folatos (EDF). Esta fórmula fue aplicada a los folatos sanguíneos provenientes de estudios chilenos transformando $\mathrm{nmol} / \mathrm{L}$ a $\mathrm{mg} / \mathrm{L}$ a través de la siguiente fórmula:

$$
\mathrm{mg} / \mathrm{L}=\frac{\mathrm{nmol} / \mathrm{L}}{2,266}
$$

Los valores promedio y la desviación estándar de la ingesta diaria de EDF obtenidos al aplicar la fórmula fueron utilizados para estimar el porcentaje de población con mayor riesgo en el período pre-fortificación y post-fortificación. Para efecto de cálculos, se asumió una distribución gaussiana normal de los requerimientos de folatos ${ }^{30}$. A partir de valores de ingesta de EDF y considerando los 
valores z de la distribución, se estimó el porcentaje de las poblaciones con mayor probabilidad de tener ingestas superiores al requerimiento promedio estimado (EAR) para folatos $(320 \mu \mathrm{g} / \mathrm{d})$ e ingestas cercanas al UL ${ }^{21}$. Como la ingesta de folatos naturales no es equivalente al ácido fólico y considerando que el UL está definido para AF, éste fue convertido en EDF utilizando un factor de conversión de 1,7 $(\mathrm{EDF}=1.000 \mathrm{mg} \times 1,7)^{16}$.

\section{Resultados}

En la Tabla 1 se observa que la mediana del contenido de AF en harinas varía de $1,9 \mu \mathrm{g} / \mathrm{kg}$ en el año 2005 y $1,5 \mathrm{mg} / \mathrm{kg}$ en 2007. Estos niveles representan entre 13,6 y $31 \%$ menos que lo es- tablecido en la norma chilena. Durante los años 2005, 2006 y 2007 no se detectó AF en cerca del $10 \%$ de las muestras. Por otra parte, alrededor del $30 \%$ tienen contenidos elevados $(>2,5 \mathrm{mg} / \mathrm{kg})$. En el año 2008 la mediana de AF en harinas fue $50 \%$ menor a lo establecido $(1,1 \mathrm{mg} / \mathrm{kg})$ y más baja que los años anteriores. En este último año, 20\% de las muestras no contenían AF y $10 \%$ contenía niveles elevados $(>2,6 \mathrm{mg} / \mathrm{kg})$.

La ingesta estimada de AF proveniente del consumo aparente de pan aplicando los niveles de fortificación encontrados en harinas y considerando los percentiles 20, 50 y 95 se muestran en la Tabla 2. Se observa que entre los años 2005 a 2007, un consumo de 2 a 3 panes diarios elaborados con harinas con niveles de AF sobre el P95 permitirían alcanzar ingestas superiores al UL. Durante el año

Tabla 1. Contenido de ácido fólico $(\mathrm{mg} / \mathrm{kg})$ en harina de trigo según percentiles (P). Chile 2005-2008

\begin{tabular}{|lllllllllllll|}
\hline Año & $\mathbf{n}$ & $\mathbf{P 1 0}$ & $\mathbf{P 2 0}$ & $\mathbf{P 3 0}$ & $\mathbf{P 4 0}$ & $\mathbf{P 5 0}$ & $\mathbf{P 6 0}$ & $\mathbf{P 7 0}$ & $\mathbf{P 8 0}$ & P90 & P95 & P97 \\
2005 & 338 & 0 & 0,59 & 1,21 & 1,51 & 1,9 & 2,2 & $2,5^{*}$ & $3^{*}$ & $4^{*} 3^{*}$ & $5,9^{*}$ & $7,4^{*}$ \\
2006 & 391 & 0,2 & 0,65 & 1,01 & 1,36 & 1,61 & 1,99 & $2,4^{*}$ & $3,06^{*}$ & $5,03^{*}$ & $9,8^{*}$ & $15,9^{*}$ \\
2007 & 279 & 0,1 & 0,58 & 0,99 & 1,27 & 1,51 & 1,77 & $2,3^{*}$ & $2,8^{*}$ & $4,8^{*}$ & $8,6^{*}$ & $10,1^{*}$ \\
2008 & 243 & 0 & 0 & 0,3 & 0,68 & 1,1 & 1,5 & 1,8 & 2 & $2,6^{*}$ & $3,2^{*}$ & $3,7^{*}$ \\
\hline
\end{tabular}

Fuente: Calculado a partir de la base de datos proporcionada por el Instituto de Salud Pública (ISP). *Valores sobre la norma chilena de fortificación de harina de trigo con ácido fólico $(2,2 \mathrm{mg} / \mathrm{kg})$.

Tabla 2. Estimación de ingesta de ácido fólico AF ( $\mu \mathrm{g} / \mathrm{dí} a)$ en adultos según consumo aparente de pan y distintos niveles de fortificación de harinas

\begin{tabular}{|c|c|c|c|c|c|c|c|c|c|c|c|}
\hline \multirow[b]{2}{*}{ Percentiles } & \multicolumn{3}{|c|}{ Año 2005} & \multicolumn{3}{|c|}{ Año 2006} & \multicolumn{3}{|c|}{ Año 2007} & \multicolumn{2}{|c|}{ Año 2008* } \\
\hline & P20 & P50 & P95 & P20 & P50 & P95 & P20 & P50 & P95 & P50 & P95 \\
\hline $\begin{array}{l}\text { A. Fólico } \\
\text { Harinas } \\
(\mu \mathrm{g} / 100 \mathrm{~g})\end{array}$ & 60 & 190 & 590 & 70 & 160 & 980 & 58 & 151 & 860 & 110 & 320 \\
\hline $\begin{array}{l}\text { A. Fólico } \\
\text { Pan } \\
(\mu \mathrm{g} / 100 \mathrm{~g})\end{array}$ & 52 & 165 & 513 & 61 & 139 & 853 & 50 & 131 & 748 & 96 & 278 \\
\hline 1 pan & 52 & 165 & 513 & 61 & 139 & 853 & 50 & 131 & 748 & 96 & 278 \\
\hline 2 panes & 104 & 331 & 1.027 & 122 & 278 & 1.705 & 101 & 263 & 1.496 & 191 & 557 \\
\hline 3 panes & 157 & 496 & 1.540 & 183 & 418 & 2.558 & 151 & 394 & 2.245 & 287 & 835 \\
\hline 4 panes & 209 & 661 & 2.053 & 244 & 557 & 3.410 & 202 & 525 & 2.993 & 383 & 1.114 \\
\hline 5 panes & 261 & 827 & 2.567 & 305 & 696 & 4.263 & 252 & 657 & 3.741 & 479 & 1.392 \\
\hline
\end{tabular}

${ }^{*}$ Año 2008 P20 = 0. Valores con negrita = Ingestas estimadas de AF ( $\mu \mathrm{g} /$ día) superiores al UL (1.000 $\left.\mu \mathrm{g}\right)$. 
2008 y en correspondencia con el menor contenido de AF en harinas, el UL se alcanzaría con mayores consumos de pan.

En la Figura 1 se presenta una estimación de la ingesta de $\mathrm{AF}$ en niños entre 8 y 13 años según NSE considerando sólo consumo de pan (gramos/ día) según P 20,50 y 75 y asumiendo que la harina utilizada cumple con el nivel de fortificación establecido $(2,2 \mathrm{mg} / \mathrm{kg})$. Se observa que los niños de nivel socioeconómico bajo, con consumos diarios de pan sobre $\mathrm{P} 75$ podrían tener ingestas cercanos al UL $(600 \mu \mathrm{g} / \mathrm{d})^{21}$.

Las ingestas promedio de EDF y sus desviaciones estándar en distintos grupos de población fueron estimadas a partir de la ecuación de Quinlivan \& Gregory ${ }^{28,29}$ y comparadas con el EAR y el UL establecidos para folatos (Tabla 3). Durante la pre-fortificación, sólo $42,7 \%$ de las mujeres tenía ingestas superiores al EAR, aumentando a 99\% en el período post-fortificación, con ingestas promedios 3,5 veces superior al EAR y donde $2,3 \%$ tenía ingestas cercanas al UL. Los datos del estudio de ancianos ${ }^{24}$, permiten estimar que $82 \%$ presentaba ingesta de EDF superiores al EAR. En este mismo grupo se observa un aumento durante la etapa post-fortificación, encontrándose que el $100 \%$ tenía ingestas superiores al EAR. Considerando los valores de folatos séricos de un estudio

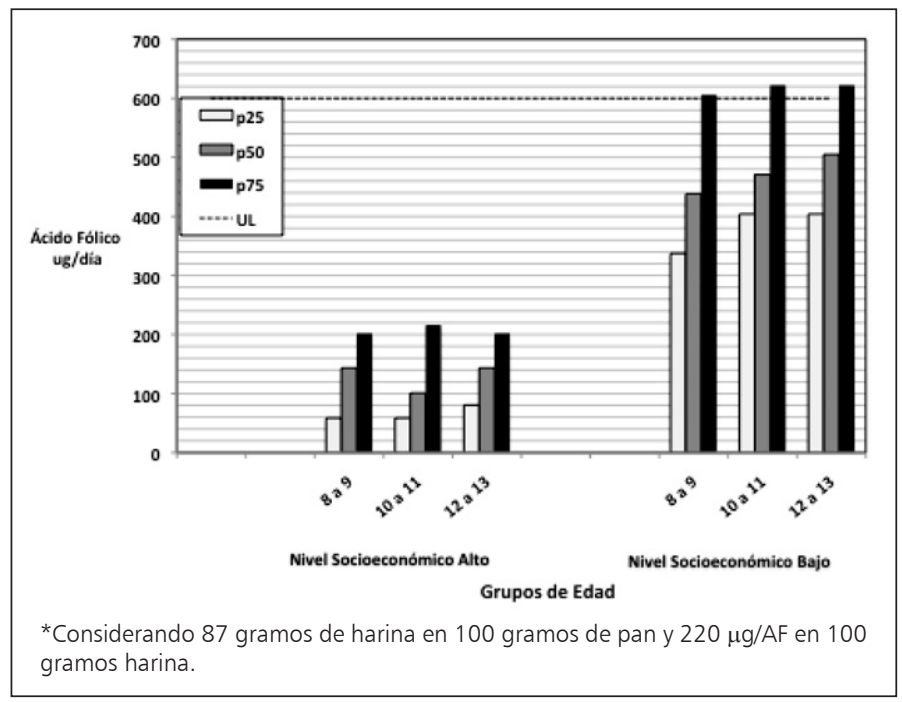

Figura 1. Consumo aparente de ácido fólico

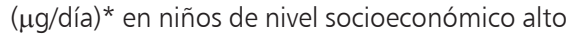
y bajo según grupos de edad y percentiles de consumo de pan (gramos/día).

Tabla 3. Estimación de ingesta de Equivalentes Dietarios de Folatos ( $\mu \mathrm{g} / \mathrm{d}$ ía) y de poblaciones con ingestas sobre Requerimiento Promedio Estimado y Nivel Máximo Recomendado (\%)

\begin{tabular}{|c|c|c|c|c|}
\hline Grupos estudiados & $\begin{array}{l}\text { Nivel de folatos } \\
\text { séricos promedio } \\
(\mu \mathrm{g} / \mathrm{L})\end{array}$ & $\begin{array}{c}\text { Ingesta promedio } \\
\text { EDF* } \\
\text { ( } \mu \text { g/día) }\end{array}$ & $\begin{array}{c}\text { Población } \\
\text { (\%) } \\
\text { Ingestas } \\
>\text { EAR* }^{*}\end{array}$ & $\begin{array}{l}\text { Población } \\
\text { (\%) } \\
\text { Ingestas } \\
\text { > UL* }\end{array}$ \\
\hline Mujeres. Pre-fortificación** & 4,3 & 286,1 & 42 & 0 \\
\hline Mujeres. Post-fortificación*** & 16,4 & $1.123,1$ & 99,7 & 2,3 \\
\hline Adultos Mayores. Pre-fortificaciónº & 7,1 & 483,9 & 81,6 & 0 \\
\hline Adultos Mayores. Post-fortificaciónº & 14,4 & 986,1 & 100 & 0 \\
\hline Hombres Adultos. Post-fortificación ${ }^{\circ o}$ & 15,3 & $1.043,9$ & 97 & 6 \\
\hline
\end{tabular}

*Equivalente Dietario de Folato $=$ EDF; Requerimiento Promedio Estimado = EAR; Nivel Máximo Estimado $=$ UL. **Hertrampf E, 2003 ${ }^{15,19}$. ***Hertrampf E, 2003 ${ }^{15,19}$. oHirsch S, 200117,20. ${ }^{\circ}$ Hirsh S, $2005^{18}$. 

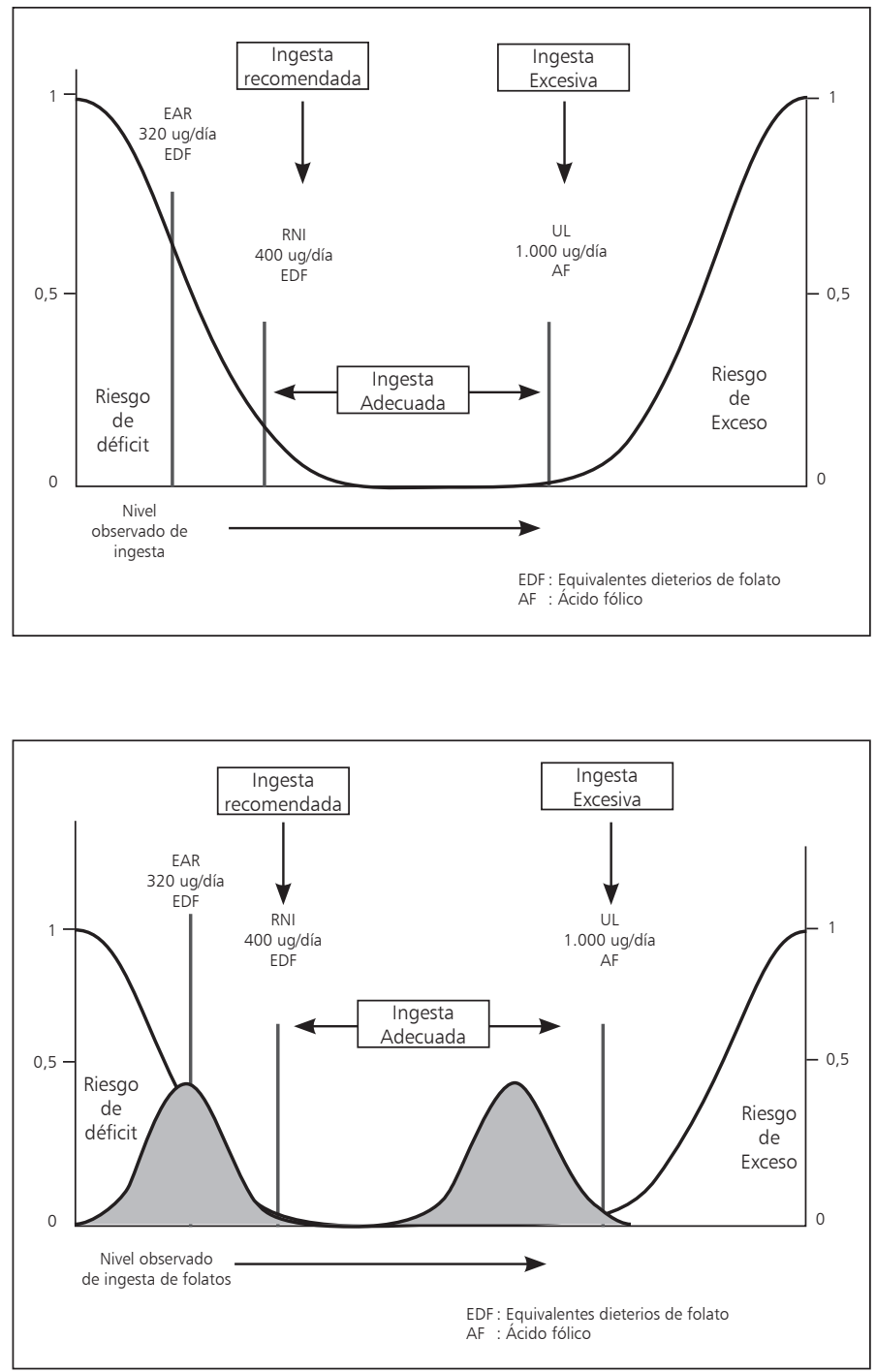

Figura 2A. Modelo para estimar la ingesta de folatos ( $\mu \mathrm{g} / \mathrm{d}$ ía) asumiendo una distribución Gaussiana del Requerimiento.
Figura 2B. Efecto de la fortificación de la harina de trigo con ácido fólico en la ingesta de folatos en un grupo de mujeres (edad fértil) pre-fortificación y post-fortificación. desarrollado con posterioridad (año 2008) ${ }^{25}$, se puede observar que la ingesta de EDF estimada en hombres adultos era 3,2 veces superior al EAR y donde $6 \%$ tenía ingestas superiores al UL.

En la Figura 2A se representa un modelo para estimar riesgo por exceso de folatos asumiendo una distribución gaussiana del requerimiento.

En la Figura 2B se utilizan los valores estimados de EDF en mujeres presentado en la Tabla 3, para mostrar en forma gráfica, el aumento de la ingesta de folatos derivado de la fortificación de la harina. En esta figura se puede observar el desplazamiento de la curva de ingesta hacia la derecha, mostrando el porcentaje de la población con ingestas superiores al UL $(2,3 \%)$.

\section{Discusión}

Los folatos cumplen funciones en la metilación biológica y en la síntesis de ácidos nucleicos que explican sus beneficios, pero también sus probables riesgos ${ }^{21}$. Los folatos de los alimentos difieren de la estructura química del AF utilizado en la fortificación de alimentos, lo cual determina una metabolización diferente ${ }^{20}$. Algunos estudios sugieren que la reducción enzimática y la metila- 
ción del AF durante la absorción en el intestino es dosis dependiente, saturándose los mecanismos normales de absorción sobre $200-266 \mu \mathrm{g} / \mathrm{d}$ y determinando la presencia de AF sin metabolizar en el suero que aumentaría cuando las dosis se repiten $^{31,32}$. El mayor riesgo en salud dependería no sólo de su nivel de ingesta, sino también, de la extensión en el tiempo de su consumo. Esto ha determinado que países como Gran Bretaña posterguen la fortificación ${ }^{33}$.

El objetivo de este estudio ha sido evaluar el caso de la fortificación de la harina de trigo con $\mathrm{AF}$ en Chile y la existencia de poblaciones en riesgos por ingestas excesivas de folatos. Para esto se ha analizado el contenido de AF en muestras de harina que procesa el sistema nacional de vigilancia chileno. En general, se observa una gran variabilidad del contenido de AF, con 10 a $20 \%$ de harinas sin $\mathrm{AF}$, por otra parte se encuentra 10 a 30\% de muestras con niveles superiores a la norma. Esto puede generarse por una granulometría inadecuada de la mezcla vitamínica que limita la homogeneización, por un mezclador inadecuado o por el tiempo de mezclado. Los niveles elevados pueden corresponder también a una sobredosificación para asegurar el cumplimiento del mínimo establecido, sugiriendo la existencia de dificultades para asegurar la calidad del producto. Otros factores a considerar es el método de toma de muestras y de análisis, aún cuando la técnica ha sido adecuadamente validada ${ }^{34}$. Los niveles de AF durante el año 2008, con una mediana de 1,1 $\mathrm{mg} / \mathrm{kg}$, representan un valor $50 \%$ más bajo que lo normado y observado en años anteriores. Esto podría corresponder a una adecuación efectuada por la industria siguiendo recomendaciones entregadas en seminarios técnicos del MINSAL ${ }^{24}$. Sin embargo, la caída de la mediana en 50\% sugiere la existencia de dificultades fabriles que debieran ser corregidas.

Un aspecto a analizar es la oportunidad ${ }^{35}$ en que se estableció el monitoreo de la fortificación en Chile. Esto ocurrió cuatro años después de iniciada la fortificación (año 2005), situación que no ha permitido conocer los niveles de $\mathrm{AF}$ durante los primeros años (2000-2004), limitando la identificación de riesgos y retrasando adecuaciones o modificaciones. Sin embargo, la fortificación con AF en Chile se considera una intervención exitosa que cumplió con los objetivos planteados, reducir la prevalencia de RN con DTN, mostrando que aunque no se conocían los valores en harinas durante los primeros años, la fortificación se estaba desarrollando. Datos de 9 hospitales de Santiago muestran el descenso en el número de casos con DTN (18 a 7,4/10.000 nacimientos en 1999 y 2006 respectivamente) ${ }^{26}$. Se ha descrito recientemente que el déficit de vitamina $B_{12}$ sería un factor determinante de algún porcentaje de los DTN $^{36}$, mostrando que una reevaluación y ajuste en los niveles de AF es posible, lo cual permitiría mantener la protección en niños, pero limitando riesgos por exceso en otros grupos.

Como la dosis, el tiempo y el momento de la suplementación ${ }^{13-15}$ son factores que determinan riesgos, se ha descrito que dependiendo del nivel de ingesta se establecería una curva de riesgos en forma de $\mathrm{U}$, donde bajas dosis contribuirían al desarrollo de cáncer de colon, mientras que altas dosis ( $1 \mathrm{mg} \mathrm{AF} /$ día) e ingestas prolongadas ( $>3$ años) contribuiría a mayores riesgos en portadores de lesiones neoplásicas incipientes ${ }^{37}$. Al parecer, el cáncer sobrerregularía los receptores de folatos para responder a las necesidades aumentadas de nucleótidos necesarios para la síntesis de $\mathrm{ADN}^{38}$. Por otra parte, los beneficios de una mayor ingesta para prevenir cáncer de colon se describen en sujetos sanos, consumidores de alcohol y portadores del polimorfismo C677T en su variedad TT ${ }^{39,40}$.

Algunos estudios describen también un riesgo 5 veces mayor para deterioro neurológico y cognitivo en ancianos asociado a niveles elevados de folatos sanguíneos ( $>59 \mathrm{nmol} / \mathrm{L}$ ) y bajos niveles de vitamina $\mathrm{B}_{12}(<148 \mathrm{pmol} / \mathrm{L})$, demostrando la interrelación del metabolismo de los folatos con otras vitaminas ${ }^{16,17}$. Estos hallazgos sugieren la necesidad de contar probablemente, con UL diferenciados por grupo de edad y condición genética, entre otras variables, consideraciones que podrían dificultar el establecimiento de niveles adecuados y límites seguros de AF para fortificaciones masivas.

Los datos de este estudio muestran que la ingesta de $\mathrm{AF}$ en adultos, estimada a través de consumo aparente de pan, podría determinar la existencia de grupos con una mayor probabilidad de riesgos por ingestas cercanas al UL. La estimación de ingesta de fólico provenientes de consumo de pan en niños de menores recursos muestra que aquellos con mayores consumos y considerando niveles de fólico en harina según la norma chilena podrían tener ingestas excesivas, situación que debiera verificarse en futuros estudios. 
Estas estimaciones tienen sus limitaciones porque fueron realizadas considerando sólo consumo aparente de pan, aún cuando en Chile existe un consumo frecuente de otros productos fortificados preparados con harina de trigo ${ }^{41}$ pudiendo esperarse ingestas superiores. El consumo de folatos y ácido fólico deben ser evaluados considerando la gran variabilidad del contenido de AF en harinas (Tabla 1) para limitar eventuales riesgos.

La ecuación propuesta por Quinlivan \& Gregory si bien corresponde a una forma indirecta para establecer consumo de folatos y no reemplaza la determinación del consumo por encuestas o de medición de indicadores bioquímicos, resulta ser una herramienta útil para estimar la ingesta de EDF diarios y los riesgos por déficit o exceso a partir de valores de folatos séricos ${ }^{28,29}$. Esta corresponde a una regresión linear, aún cuando es esperable una curva de saturación, se basó en estudios que reportaban consumo de folatos y niveles séricos, validados con encuestas de consumo, con una alta correlación, siendo utilizada en otros estudios para estimar ingesta de folatos ${ }^{42}$. En este estudio se estima que el $85 \%$ de los ancianos durante la pre-fortificación presentaban niveles de folatos sanguíneos sobre el EAR, niveles que podrían relacionarse con el AF aportado por un alimento fortificado del Programa Nacional de Alimentación del Adulto Mayor ${ }^{43}$. En mujeres, donde no existía entrega de alimentos fortificados, sólo $42 \%$ presentaba ingestas superiores al EAR justificando la necesidad de la intervención.

Las Figuras 2A y B muestran en forma gráfica el cambio en la ingesta de folatos después de la fortificación, destacando que ya en los primeros años de fortificación (datos del año 2003) el 2,6\% de las mujeres presentaba ingestas superiores al UL.

Otra limitación se genera por los niveles de folatos sanguíneos utilizados en el análisis que corresponden preferentemente a valores de los primeros años de fortificación. Considerando que el consumo de harina fortificada ha sido permanente y que un porcentaje presenta de ella presenta niveles de fortificación sobre lo establecido, los actuales valores de folatos séricos debieran ser más altos, aumentando la probabilidad de riesgos, como muestran los datos de folatos séricos en hombres (año 2008) que permiten estimar ingestas promedio de 1044 EDF/día (Tabla 3).

Los resultados de este estudio muestran la necesidad de realizar una evaluación de riesgos integrada de esta intervención nutricional en Chile, considerando los beneficios y los riesgos. Esto permitiría adecuar el nivel de fortificación en harinas considerando que existe un porcentaje de mujeres, niños y adultos con ingestas de ácido fólico cercanas al UL. El principio de beneficencia implicaría tomar las mejores medidas para optimizar los beneficios pero minimizando los riesgos ${ }^{42}$, reduciendo la sobreexposición innecesaria en algunos grupos, pero asegurando los beneficios en embarazadas.

\section{Referencias}

1. Gobierno de Chile. Ministerio de Salud. La carga de enfermedad en Chile 1996. Disponible en: http://epi. minsal.cl/epi/html/sdesalud/carga/Inffin-carga-enf.pdf [Consultado el 15 diciembre de 2009].

2. Nazer JH, Aravena TC, Cifuentes LO. Malformaciones congénitas en Chile. Un problema emergente (período 1995-1999). Rev Med Chile 2001; 129: 895-904.

3. Gobierno de Chile. Ministerio de Salud. Norma Técnica para la Fortificación de la Harina de trigo con Vitaminas y Minerales. Diciembre de 1999.

4. Gobierno de Chile. Ministerio de Salud. Reglamento Sanitario de los Alimentos. Disponible en: http:// www.redsalud.gov.cl/portal/url/page/minsalcl/g_ proteccion/g_alimentos/reglamento_sanitario_alimentos.html [Consultado el 25 de julio de 2009].

5. Smithells RW, Sheppard S, Schorah CJ, Seller MJ, Nevin NC, Harris R, et al. Apparent prevention of neural tube defects by periconceptional vitamin supplementation. Arch Dis Child 1981; 56: 911-8.

6. Laurence Km, James N, Miller MH, Tennant GB, Campbell $\mathrm{H}$. Double-blind randomised controlled trial of folate treatment before conception to prevent recurrence of neural-tube defects. BMJ (Clin Res Ed) 1981; 282 (6275): 1509-11.

7. Werler MM, Shapiro S, Mitchell AA. Periconceptional folic acid exposure and risk of occurrent neural tube defects. JAMA 1993; 269 (10): 1257-61.

8. Shaw Gm, Schaffer D, Velie Em, Morland K, Harris JA. Periconceptional vitamin use, dietary folate, and the occurrence of neural tube defects. Epidemiology 1995; 6: 219-26.

9. Espinosa F. SISVAN de alimentos Índices. Consumo de alimentos y cambios de hábitos alimentarios. INTA. Universidad de Chile. Santiago: 1998.

10. Hertrampf E, Cortés F. National food-fortification program with folic acid in Chile. Food Nutr Bull 2008; 29 
(2 Suppl): S231-7.

11. Llanos A, Hertrampf E, Cortés F, Pardo A, Grosse SD, Uauy R. Cost-effectiveness of a folic acid fortification program in Chile. Health Policy 2007; 83 (2-3): 295-303.

12. Food And Agriculture Organization Of The United Nations And World Health Organization (FAO-WHO). A Model for Establishing Upper Levels of Intake for Nutrients and Related Substances Report of a Joint FAO/WHO Technical Workshop on Nutrient Risk Assessment. WHO Headquarters, Geneva, Switzerland 2-6 May 2005.

13. Kim YI. Folate and colorectal cancer: An evidence-base critical review. Mol Nutr Food Res 2007; 51: 267-92.

14. Van Guelpen B, Hultdin J, Johansson I, Hallmans G, Stenling R, Riboli E, et al. Low folate levels may protect against colorectal cancer. Gut 2006; 55: 1387-9.

15. Sanjoaquin MA, Allen N, Couto E, Roddam AW, Key TJ. Folate intake and colorectal cancer risk: A metaanalytical approach, Int J Cancer 2005; 113: 825-8.

16. Selhub J, Morris Ms, Jacques Pf, Rosenberg IH. Folatevitamin B-12 interaction in relation to cognitive impairment, anemia, and biochemical indicators of vitamin B-12 deficiency. Am J Clin Nutr 2009; 89: 702S.

17. Miller JW, Garrod MG, Allen LH, Haan MN, Green R. Metabolic evidence of vitamin B-12 deficiency, including high homocysteine and methylmalonic acid and low holotranscobalamin, is more pronounced in older adults with elevated plasma folate. Am J Clin Nutr 2009; 90: 1586-92.

18. Stolzenberg-Solomon RZ, Chang SC, Leitzmann MF, Johnson KA, Johnson C, Buys SS, et al. Folate intake, alcohol use, and postmenopausal breast cancer risk in the Prostate, Lung, Colorectal, and Ovarian Cancer Screening Trial. Am J Clin Nutr 2006; 83: 895-904.

19. Figueiredo JC, Grau MV, Haile RW, Sandler RS, Summers RW, Bresalier RS, et al. Folic acid and risk of prostate cancer: results from a randomized clinical trial. J Natl Cancer Inst 2009; 101: 432-5.

20. Food And Drug Administration (FDA). Final Rule. Food Standards: Amendment of Standards of Identity for Enriched Grain Products to Require Addition of Folic Acid. Federal Register 1996; 61: 8781-97.

21. National Research Council (NRC). Recommended Dietary Allowances. 10th ed. Washington, DC: National Academy Press, 1989.

22. Shakur YA, Rogenstein C, Hartman-Craven B, Tarasuk V, O'connor DL. How much folate is in Canadian fortified products 10 years after mandated fortification? Can J Public Health 2009; 100: 281-4.

23. Hertrampf E, Cortés F, Erickson JD, Cayazzo M, Freire W, Bailey LB, et al. Consumption of folic acid-fortified bread improves folate status in women of reproductive age in Chile. J Nutr 2003; 133: 3166-9.

24. Hirsch S, De La Maza P, Barrera G, Gattás V, Petermann $\mathrm{M}$, Bunout D. The Chilean flour folic acid fortification program reduces serum homocysteine levels and masks vitamin B-12 deficiency in elderly people. J Nutr 2002; 132: 289-91.

25. Hirsch S, Ronco AM, Guerrero-Bosagna C, De la Maza MP, Leiva L, Barrera G, et al. Methylation status in healthy subjects with normal and high serum folate concentration. Nutrition 2008; 24 (11-12): 1103-9.

26. Gobierno de Chile. Ministerio de Salud. Seminario de Fortificación de Harinas. Enero de 2008. Disponible en: http://www.redsalud.gov.cl/portal/url/page/ $\mathrm{minsalcl} / \mathrm{g} \_$proteccion/g_alimentos/prot_fortificacion. html [Consultado el 14 de diciembre de 2009].

27. Olivares S, Bustos N, Lera L, Zelada ME. Estado nutricional, consumo de alimentos y actividad física en escolares mujeres de diferente nivel socioeconómico de Santiago de Chile. Rev Med Chile 2007; 135: 71-8.

28. Quinlivan EP, Gregory JF 3rd. Effect of food fortification on folic acid intake in the United States. Am J Clin Nutr 2003; 77: 221-5.

29. Quinlivan EP, Gregory JF 3rd. Reassessing folic acid consumption patterns in the United States (1999 2004): potential effect on neural tube defects and overexposure to folate. Am J Clin Nutr 2007; 86: 1773-9.

30. Uauy-Dagach R, Hertrampf E. Food-based dietary recommendations: possibilities and limitations. In: Bowman B, Russell R, eds. Present knowledge in nutrition, $8^{\text {th }}$ ed. Washington DC: ILSI Press; 2001: 636-49.

31. Sweeney MR, Mcpartlin J, Scott J. Folic acid fortification and public health: report on threshold doses above which unmetabolised folic acid appear in serum. BMC Public Health 2007; 7: 41.

32. Sweeney MR, Mcpartlin J, Weir DG, Daly L, Scott JM. Postprandial serum folic acid response to multiple doses of folic acid in fortified bread. Br J Nutr 2006; 95: 14551.

33. Standing Advisory Committee On Nutrition. Folate and disease prevention. London, United Kingdom: The Stationary Office, 2006. Disponible en: http://www.sacn. gov.uk/reports_position_statements/reports/report_on_ folate_and_disease_prevention.html [Consultado el 1 de junio de 2010] .

34. Gobierno de Chile. Instituto de Salud Pública. Programa de Fortificación de Harinas. Disponible en: http://www. ispch.cl/programa-de-fortificacion-de-harinas [Consultado el 1 de junio de 2010] .

35. Allen L, Benoist B, Dary O, Hurrell R. Guidelines on food fortification with micronutrients. World Health 
Organization and Food and Agriculture Organization of the United Nations 2006.

36. Li F, Watkins D, Rosenblatt DS. Vitamin B(12) and birth defects. Mol Genet Metab 2009; 98 (1-2): 166-72.

37. Cole BF, Baron JA, Sandler RS, Haile RW, Ahnen DJ, Bresalier RS, et al. Polyp Prevention Study Group. Folic acid for the prevention of colorectal adenomas: a randomized clinical trial. JAMA 2007; 297 (21): 23519.

38. Mason JB. Folate, cancer risk, and the Greek god, Proteus: a tale of two chameleons. Nutrition Rev 2009; 67: 206-12.

39. Bailey LB. Folate, methyl-related nutrients, alcohol, and the MTHFR 677C-->T polymorphism affect cancer risk: intake recommendations. J Nutr 2003; 133 (11 Suppl 1): 3748S-53S.

40. Kim YI. 5,10-Methylenetetrahydrofolate reductase polymorphisms and pharmacogenetics: A new role of single nucleotide polymorphisms in the folate metabolic pathway in human health and disease. Nutr Rev 2005; 63: 398-407.

41. Muñoz O, Bastías JM, Araya M, Morales A, Orellana C, Rebolledo R, et al. Estimation of the dietary intake of cadmium, lead, mercury, and arsenic by the population of Santiago (Chile) using a Total Diet Study. Food Chem Toxicol 2005; 43: 1647-55.

42. Dary O. Establishing safe and potentially efficacious fortification contents for folic acid and vitamin B12. Food Nutr Bull 2008; 29 (2 Suppl): S214-24.

43. Gobierno de Chile. Ministerio de Salud. Programa de Alimentación Complementaria del Adulto Mayor. PACAM. Disponible en: http://www.redsalud.gov.cl/portal/ url/page/minsalcl/g_proteccion/g_alimentos/prot_pan. html [Consultado el 23 diciembre de 2009]. 